$>$ De nombreux facteurs de risque professionnels, notamment le stress au travail, sont associés à un risque cardiovasculaire élevé. Le stress professionnel présente diverses composantes, en particulier la tension entre les contraintes imposées par le travail et la latitude pour y faire face (modèle de Karasek), et le déséquilibre entre efforts consentis et récompenses reçues (modèle de Siegrist). II est établi que l'exposition chronique au stress professionnel est un facteur indépendant de risque de pathologie coronarienne ischémique, et le pourcentage de cas d'infarctus du myocarde induits par le stress professionnel est d'environ $3,5 \%$, ce qui correspond pour la France, à 3400 à 4000 cas chaque année. Divers mécanismes sont susceptibles d'expliquer le rôle du stress professionnel, mais ils restent encore largement méconnus. <

\section{Le travail \\ stressant et \\ le coeur ne font \\ pas bon ménage}

Marcel Goldberg ${ }^{1,2}$, Marie Zins ${ }^{1,2}$

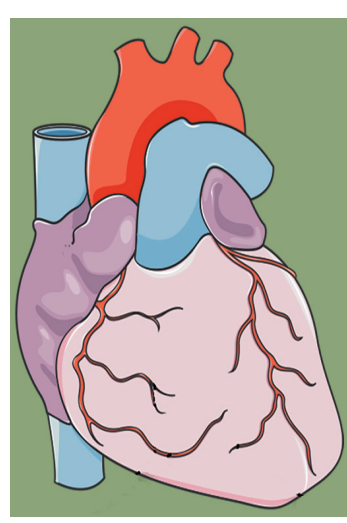

${ }^{1}$ Unité mixte Cohortes

épidémiologiques

en population,

UMS 11 Inserm-UVSQ, France.

${ }^{2}$ Université René Descartes,

Paris, France. 16, avenue

Paul Vaillant Couturier,

94800 Villejuif, France.

marcel.goldberg@inserm.fr

marie.zins@inserm.fr

reçues, le stress professionnel n'est pas l'apanage du cadre surmené : ce sont, de fait, avant tout (et de loin) les employés et ouvriers qui sont les plus exposés à ce type de facteurs [6]. Depuis les premiers travaux de Karasek au début des années 1980 [7], d'innombrables études concernant le stress au travail et le risque d'infarctus du myocarde ont été publiées.

De nombreux travaux ont mis en évidence depuis longtemps le rôle délétère de certaines conditions de travail pour le système cardiovasculaire. Ainsi, le bruit et le travail de nuit [1], certaines expositions à des produits chimiques (le monoxyde de carbone, le disulfure de carbone et divers solvants, les hydrocarbures halogénés, la nitroglycérine et les nitrates [2]), la chaleur [3], les longs horaires de travail ou la conduite automobile [4], sont des facteurs de risque établis de cardiopathies ischémiques. II en est, paradoxalement, de même de la charge de travail physique [5], alors qu'au contraire l'activité physique de loisir est un facteur protecteur pour le risque cardiovasculaire.

Cependant, ces conditions de travail et expositions professionnelles concernent pour l'essentiel les travailleurs manuels. II n'en est pas de même de ce que les spécialistes appellent les «facteurs psychosociaux au travail », plus communément dénommés «stress professionnel ». Ils concernent en effet toutes les catégories de travailleurs, même si, contrairement aux idées

Vignette (Photo @ Angelo Parini).

\section{Le stress professionnel : de quoi parle-t-on?}

Les deux principaux instruments permettant d'évaluer le stress au travail sont ceux élaborés respectivement par Karasek et Theorell [7] et Siegrist [8].

\section{Le modèle de Karasek}

Le modèle de Karasek, le plus ancien, repose sur le «Job Content Questionnaire» (JCP), composé d'une trentaine de questions (Encadré 1) qui permettent de calculer des scores sur les trois principales dimensions de l'environnement psychosocial au travail: la demande psychologique (définie par la charge psychologique associée à l'accomplissement des tâches, aux tâches imprévues, aux interruptions, aux demandes contradictoires, etc.), la latitude décisionnelle (qui comprend à la fois l'autonomie ou contrôle, et la possibilité d'utiliser et de développer ses compétences et qualifications) et le soutien social (défini par l'aide et la reconnaissance des collègues et du supérieur hiérarchique) qui a été ajouté un peu plus tard au modèle. Les scores calculés permettent de définir, selon 


\section{Job Content Questionnaire de Karasek}

\begin{tabular}{|c|c|c|c|c|}
\hline & & & & \\
\hline & $\begin{array}{l}\text { Fortement } \\
\text { en } \\
\text { désaccord }\end{array}$ & $\begin{array}{c}\varepsilon_{n} \\
\text { désaccord }\end{array}$ & D'accord & $\begin{array}{l}\text { Tout à fait } \\
\text { d'accord }\end{array}$ \\
\hline l- Mon travail nécessite que j'apprenne des choses nouvelles & $\square_{1}$ & $\square_{2}$ & $\square_{3}$ & $\square_{4}$ \\
\hline 2 - Mon travail nécessite un niveau élévé de qualifications & $\square_{1}$ & $\square_{2}$ & $\square_{3}$ & $\square_{4}$ \\
\hline 3 - Dans mon travail, je dois faire preuve de créativité & $\square_{1}$ & $\square_{2}$ & $\square_{3}$ & $\square_{4}$ \\
\hline 4 - Mon travail consiste à refaire toujours les mêmes choses & $\square_{1}$ & $\square_{2}$ & $\square_{3}$ & $\square_{4}$ \\
\hline 7 - Au travail, j'ai l'opportunité de faire plusieurs choses différentes & $\square_{1}$ & $\square_{2}$ & $\square_{3}$ & $\square_{4}$ \\
\hline 9 - Au travail, j'ai la possibilité de développer mes habiletés personnelles & $\square_{1}$ & $\square_{2}$ & $\square_{3}$ & $\square_{4}$ \\
\hline 6 - Mon travail me permet de prendre des décisions de façon autonome & $\square_{1}$ & $\square_{2}$ & $\square 3$ & $\square_{4}$ \\
\hline 5 - J'ai la liberté de décider comment je fais mon travail & $\square_{1}$ & $\square_{2}$ & $\square 3$ & $\square_{4}$ \\
\hline 8 - J'ai passablement d'influence sur la façon dont les choses se passent à mon travail & $\square$ l & $\square_{2}$ & $\square 3$ & $\square_{4}$ \\
\hline 10 - Mon travail exige d'aller très vite & $\square_{1}$ & $\square_{2}$ & $\square 3$ & $\square_{4}$ \\
\hline 11 - Mon travail exige de travailler très fort mentalement & $\square$ l & $\square_{2}$ & $\square 3$ & $\square_{4}$ \\
\hline 12 - On ne me demande pas de faire une quantité excessive de travail & $\square_{1}$ & $\square_{2}$ & $\square_{3}$ & $\square_{4}$ \\
\hline 13 - J'ai suffisamment de temps pour faire mon travail & $\square_{1}$ & $\square_{2}$ & $\square_{3}$ & $\square_{4}$ \\
\hline 14 - Je ne reçois pas de demandes contradictoires de la part des autres & $\square_{1}$ & $\square_{2}$ & $\square_{3}$ & $\square_{4}$ \\
\hline 15 - Mon travail m’oblige à me concentrer intensément pendant de longues périodes & $\square_{1}$ & $\square_{2}$ & $\square_{3}$ & $\square_{4}$ \\
\hline $\begin{array}{l}16 \text { - Ma tâche est souvent interrompue avant que je ne l'ai terminée, je dois alors } \\
\text { y revenir plus tard }\end{array}$ & $\square_{1}$ & $\square_{2}$ & $\square_{3}$ & $\square_{4}$ \\
\hline 17 - Mon travail est très souvent mouvementé & $\square_{1}$ & $\square_{2}$ & $\square 3$ & $\square_{4}$ \\
\hline $\begin{array}{l}18 \text { - Je suis souvent ralenti dans mon travail parce que je dois attendre que les } \\
\text { autres aient terminé le leur }\end{array}$ & $\square_{1}$ & $\square_{2}$ & $\square_{3}$ & $\square_{4}$ \\
\hline 19 - Mon chef se soucie du bien-être des travailleurs qui sont sous sa supervision & $\square$ l & $\square_{2}$ & $\square 3$ & $\square_{4}$ \\
\hline 20 - Mon chef prête attention à ce que je dis & $\square$ l & $\square_{2}$ & $\square 3$ & $\square_{4}$ \\
\hline 21 - Mon chef a une attitude hostile ou conflictuelle envers moi & $\square$ l & $\square_{2}$ & $\square 3$ & $\square_{4}$ \\
\hline 22 - Mon chef facilite la réalisation du travail & $\square_{1}$ & $\square_{2}$ & $\square 3$ & $\square_{4}$ \\
\hline 23 - Mon chef réussit à faire travailler les gens ensemble & $\square_{1}$ & $\square_{2}$ & $\square 3$ & $\square_{4}$ \\
\hline 24 - Les gens avec qui je travaille sont qualifiés pour les tâches qu'ils accomplissent & $\square$ l & $\square_{2}$ & $\square 3$ & $\square_{4}$ \\
\hline 25 - Les gens avec qui je travaille s'intéressent personnellement à moi & $\square_{1}$ & $\square_{2}$ & $\square_{3}$ & $\square_{4}$ \\
\hline $\begin{array}{l}26 \text { - Les gens avec qui je travaille ont des attitudes hostiles ou conflictuelles envers } \\
\text { moi }\end{array}$ & $\square_{1}$ & $\square_{2}$ & $\square 3$ & $\square_{4}$ \\
\hline 27 - Les gens avec qui je travaille sont amicaux & $\square$ l & $\square_{2}$ & $\square 3$ & $\square_{4}$ \\
\hline 28 - Les gens avec qui je travaille s'encouragent mutuellement à travailler ensemble & $\square$ l & $\square_{2}$ & $\square 3$ & $\square_{4}$ \\
\hline 29 - Les gens avec qui je travaille facilitent la réalisation du travail & $\square_{1}$ & $\square_{2}$ & $\square_{3}$ & $\square_{4}$ \\
\hline
\end{tabular}


l'intensité de la demande psychologique et de la latitude décisionnelle, quatre cadrans représentés dans la Figure 1 : combinaison d'une forte demande et d'une faible latitude (sujet stressé), forte demande et forte latitude (sujet actif), faible demande et faible latitude (sujet passif), et faible demande et forte latitude (sujet détendu).

L'ouvrier(e) à la chaîne ou la caissière de supermarché sont des archétypes du travail «stressé », car ils sont soumis à des fortes contraintes de travail et ne disposent pas de possibilité de les modifier ; le cadre supérieur ou les professions libérales se situent parmi les actifs (forte demande, mais possibilité d'organiser son travail). Ces caractéristiques expliquent pourquoi, contrairement aux idées reçues, ce sont les femmes qui sont le plus fréquemment exposées à un travail stressant, d'autant qu'il se surajoute au travail familial.

Selon Karasek, la combinaison d'une forte demande psychologique et d'une faible latitude décisionnelle, qui constitue le «job strain» conduit à une situation à risque, en particulier pour les maladies cardiovasculaires; l'association du job strain avec un manque de soutien social au travail définit le « iso-strain » qui a comme effet d'augmenter le risque cardiovasculaire.

\section{Le modèle de Siegrist}

Le modèle de Siegrist repose sur un autre concept: le déséquilibre entre les efforts fournis dans son travail et les récompenses obtenues en retour. II définit deux dimensions, celles des efforts (contraintes professionnelles liées au temps, aux interruptions, aux responsabilités, à la charge physique, et à une exigence forte de la tâche) et celle des récompenses (estime, perspectives de promotion et de salaire, stabilité de la situation de travail), auxquelles est ajoutée une troisième dimension définie comme le surinvestissement et qui concerne des comportements associés à un engagement excessif dans le travail. La mesure du déséquilibre efforts-récompense repose sur un questionnaire dont la version la plus récente comporte 16 items (Encadré 2) qui permettent de calculer un score d'effort et un score de récompense; le rapport du score effort sur le score récompense indique un déséquilibre lorsqu'il est supérieur à 1, d'autant plus important que la valeur est élevée.

Selon Siegrist, un déséquilibre entre des efforts élevés et des récompenses faibles conduit à une situation de détresse socio-émotionnelle, susceptible d'accroître le risque cardiovasculaire. De plus, un niveau élevé de surinvestissement serait un facteur de risque supplémentaire. Ces deux instruments existent en langue française et présentent des qualités psychométriques satisfaisantes $[9,10]$ permettant ainsi I'utilisation de questionnaires validés dans les études épidémiologiques de grande dimension et les comparaisons entre les études.

De nombreuses enquêtes ont observé des prévalences de job strain autour de $25 \%$ en France et dans divers pays, et les prévalences observées pour le déséquilibre entre efforts et récompenses sont souvent inférieures à 10 \% [6]. Les modèles de Karasek et Siegrist sont complémentaires, et leurs dimensions respectives ont montré des effets prédictifs indépendants sur divers indicateurs de santé, en particulier de santé cardiovasculaire.

Outre ces deux modèles dominants dans la littérature scientifique, d'autres aspects de l'environnement psychosocial au travail ont fait

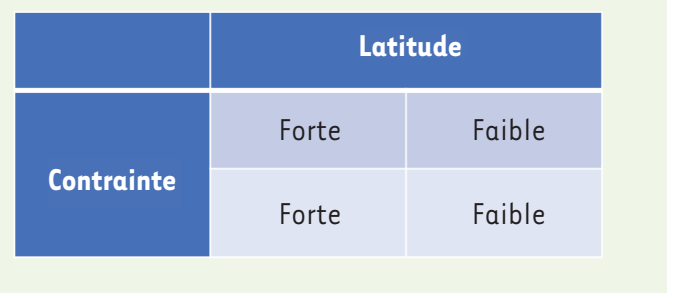

Figure 1. Les quatre situations psychosociales de travail du modèle de Karasek.

plus récemment l'objet de recherches: la violence psychologique au travail et la justice organisationnelle.

- La violence psychologique au travail est au centre de nombreuses préoccupations en milieu de travail, cette tendance s'inscrivant dans une sensibilisation accrue de nos sociétés face au problème des violences en général. En France, la notion de harcèlement moral a été introduite dans le Code du travail. Cependant, les connaissances et les données épidémiologiques restent limitées et ont concerné essentiellement les effets sur la santé mentale, et sauf omission de notre part, l'impact sur la santé cardiovasculaire n'a pas été étudié.

- La justice organisationnelle est un concept récent. Elle concerne les procédures organisationnelles et les relations sociales au travail. On distingue la justice procédurale (méthodes et mécanismes utilisés pour obtenir des résultats), la justice relationnelle (modalités par lesquelles les personnes sont traitées sur le lieu du travail en termes de relations sociales), et la justice informationnelle (modalités de communication de la hiérarchie sur les procédures et les résultats). On ne dispose actuellement que de très peu de données sur les effets sur la santé d'une mauvaise justice organisationnelle, excepté une étude dans la cohorte anglaise Whitehall montrant un risque accru de pathologie coronarienne ischémique [11].

\section{Quels effets du stress professionnel sur la pathologie coronarienne?}

La littérature épidémiologique fournit des éléments convaincants soulignant les liens entre facteurs psychosociaux au travail et divers aspects de la santé, notamment pour les affections musculo-squelettiques et les pathologies mentales, la santé perçue, la qualité de vie, ou encore l'absentéisme pour raison de santé [12-14].

Mais c'est surtout dans le domaine cardiovasculaire que les effets délétères du stress au travail ont été le plus étudiés (pour une revue récente voir [15]). Globalement, on admet aujourd'hui que les effets du stress 


\section{Questionnaire Déséquilibre efforts-récompenses de Siegrist}

\begin{tabular}{|c|c|c|c|c|}
\hline & \\
\hline & $\begin{array}{c}\text { Pas du } \\
\text { tout } \\
\text { d'accord }\end{array}$ & $\begin{array}{l}\text { Pas } \\
\text { d'accord }\end{array}$ & D'accord & $\begin{array}{c}\text { Tout } \\
\text { à fait } \\
\text { d'accord }\end{array}$ \\
\hline Je suis constamment pressé par le temps à cause d'une forte charge de travail & $\square_{1}$ & $\square_{2}$ & $\square_{3}$ & $\square_{4}$ \\
\hline Je suis fréquemment interrompu et dérangé dans mon travail & $\square_{1}$ & $\square_{2}$ & $\square_{3}$ & $\square_{4}$ \\
\hline Au cours des dernières années, mon travail est devenu de plus en plus exigeant & $\square_{1}$ & $\square_{2}$ & $\square 3$ & $\square_{4}$ \\
\hline Je reçois le respect que je mérite de mes supérieurs & $\square_{1}$ & $\square_{2}$ & $\square 3$ & $\square_{4}$ \\
\hline Mes perspectives de promotion sont faibles & $\square_{1}$ & $\square_{2}$ & $\square 3$ & $\square_{4}$ \\
\hline $\begin{array}{l}\text { Je suis en train de vivre ou je m'attends à vivre un changement indésirable dans ma } \\
\text { situation de travail }\end{array}$ & $\square_{1}$ & $\square_{2}$ & $\square_{3}$ & $\square_{4}$ \\
\hline Ma sécurité d'emploi est menacée & $\square_{1}$ & $\square_{2}$ & $\square_{3}$ & $\square_{4}$ \\
\hline Vu tous mes efforts, je reçois le respect et l'estime que je mérite à mon travail & $\square_{1}$ & $\square_{2}$ & $\square_{3}$ & $\square_{4}$ \\
\hline Vu tous mes efforts, mes perspectives de promotion sont satisfaisantes & $\square_{1}$ & $\square_{2}$ & $\square_{3}$ & $\square_{4}$ \\
\hline Vu tous mes efforts, mon salaire est satisfaisant & $\square_{1}$ & $\square_{2}$ & $\square 3$ & $\square_{4}$ \\
\hline Au travail, il m'arrive fréquemment d'être pressé par le temps & $\square_{1}$ & $\square_{2}$ & $\square 3$ & $\square_{4}$ \\
\hline Je commence à penser à des problèmes liés au travail dès que je me lève le matin & $\square_{1}$ & $\square_{2}$ & $\square 3$ & $\square_{4}$ \\
\hline $\begin{array}{l}\text { Quand je rentre à la maison, j'arrive facilement à me décontracter et à oublier tout ce } \\
\text { qui concerne mon travail }\end{array}$ & $\square_{1}$ & $\square_{2}$ & $\square_{3}$ & $\square_{4}$ \\
\hline Mes proches disent que je me sacrifie trop pour mon travail & $\square_{1}$ & $\square_{2}$ & $\square 3$ & $\square_{4}$ \\
\hline Le travail me trotte encore dans la tête quand je vais me coucher & $\square_{1}$ & $\square_{2}$ & $\square_{3}$ & $\square_{4}$ \\
\hline $\begin{array}{l}\text { Quand j'ai remis à plus tard quelque chose que j'aurai dû faire le jour même, j'ai du } \\
\text { mal à dormir le soir }\end{array}$ & $\square_{1}$ & $\square_{2}$ & $\square_{3}$ & $\square_{4}$ \\
\hline
\end{tabular}

au travail sont indépendants des autres facteurs de risque classiques de la pathologie coronarienne, avec des risques relatifs d'environ 1,2 à 1,5 .

L'étude la plus importante, publiée en 2012 dans la revue Lancet, provient du consortium européen IPD-Work (individual-participant-data meta-analysis in working populations) [16]. Le consortium regroupe plusieurs cohortes prospectives européennes (dont la cohorte française GAZEL constituée de près de 20000 agents d'EDF-GDF ${ }^{1}$ suivie depuis 1989). L'analyse a regroupé les données individuelles de 197473 participants inclus dans 13 cohortes européennes de 7 pays : Belgique, Danemark, Finlande, France, Pays-Bas, Royaume-Uni et Suisse. La moyenne d'âge des participants était de 42,3 ans et la population étudiée comportait autant de femmes que d'hommes. Le stress au travail a été évalué par le questionnaire JCP de Karasek. La proportion d'individus exposés au stress au travail était de $15,3 \%$. Sur

\footnotetext{
${ }^{1}$ Compagnie française du gaz et de l'électricité.
}

une période de 7 ans de suivi en moyenne, 2358 événements coronariens ont été recensés. Le risque relatif global sur l'ensemble des cohortes était de $1,23(1,10-$ $1,37)$; ce risque relatif était ajusté sur l'âge, le sexe, le statut socioéconomique et la répartition géographique des participants, ainsi que sur les principaux facteurs de risque classiques de l'infarctus du myocarde (tabac, alcool, activité physique, indice de masse corporelle), sur le score de Framingham (fondé sur l'âge, le cholestérol total et le HDL-cholestérol [lipoprotéines de haute densité], la pression artérielle systolique, l'utilisation d'antihypertenseurs, le tabac et le diabète). Un argument particulièrement convaincant sur le rôle du stress au travail vis-à-vis du risque d'infarctus est que, à niveau égal d'exposition au stress et aux autres facteurs de risque, on retrouve une augmentation $d u$ risque comparable dans toutes les cohortes, et ceci 
quels que soient le pays, l'âge, le sexe, ou la catégorie professionnelle, alors que jusqu'à présent, les études publiées ne concernaient que des populations restreintes qui ne permettaient pas de vérifier ces points. C'est pourquoi le rôle causal du stress au travail est aujourd'hui très largement admis, malgré l'absence d'essais randomisés (dont on ne voit d'ailleurs pas comment ils pourraient être réalisés).

La «fraction attribuable » du risque (c'est-à-dire la proportion des cas qui peuvent être attribués au stress professionnel) était de $3,4 \%$, ce qui correspond pour la France, chaque année, à environ 3400 à 4000 infarctus du myocarde qui pourraient être théoriquement évités.

\section{Quels mécanismes?}

Aux cas de pathologie coronarienne provoqués par le stress au travail, il faudrait ajouter ceux induits par d'autres facteurs de risque, tels que le tabac, l'alcool, le surpoids, les troubles du sommeil, la dépression et le manque d'exercice physique, qui sont partiellement occasionnés par le stress professionnel. En effet, un des mécanismes majeurs qui peuvent expliquer le rôle du stress professionnel vis-à-vis de la pathologie coronaire ischémique, est «indirect» car l'exposition au stress professionnel s'accompagne d'une augmentation de la fréquence des facteurs de risque classiques de cette pathologie. Ainsi dans la cohorte GAZEL, on observe que l'exposition au job strain et au déséquilibre efforts-récompenses entraînent indépendamment une augmentation du risque de troubles du sommeil et de dépression, le déséquilibre efforts-récompenses s'accompagnant de plus d'une augmentation du risque d'obésité [17]. II est intéressant de souligner que ce phénomène contribue aux inégalités sociales de santé, d'une part parce que l'exposition à ces facteurs de risque sont plus fréquents parmi les catégories sociales les moins favorisées et, d'autre part, parce que, pour des raisons qui restent largement hypothétiques, on observe que l'effet de certains facteurs de risque induits par les facteurs psychosociaux au travail sur la pathologie coronarienne, comme la dépression, est confiné aux catégories professionnelles d'ouvriers et d'employés, et n'est pas observé chez les cadres [18].

De nombreuses études ont analysé l'association du stress au travail avec des problèmes de la sphère cardiométabolique: athérosclérose, syndrome métabolique, hypertension artérielle, ainsi qu'avec l'obésité, mais ces troubles ne sont observés que de façon inconstante et lorsque c'est le cas, leur association avec le stress est faible [15]. Surtout, aucune étude longitudinale n'a pu, jusqu'à présent, confirmer la temporalité entre l'exposition au stress au travail et ces facteurs. Au total, aucun mécanisme biologique n'a pu être identifié pour expliquer le lien bien établi entre stress au travail et cardiopathies ischémiques. II semble plutôt qu'il existe des voies multiples qui peuvent constituer des éléments contribuant à l'étiologie ou susceptibles de déclencher un événement cardiovasculaire chez des sujets vulnérables. Diverses hypothèses mécanistiques ont été évoquées, sans arguments vraiment convaincants jusqu'à présent. Cependant, une publication récente a ouvert une voie nouvelle à la compréhension des mécanismes associant le stress aux pathologies cardiaques ischémiques. Cette étude où un groupe de patients, qui avaient bénéficié d'une imagerie
${ }^{18} \mathrm{~F}$-fluorodésoxyglucose $\mathrm{PET} / \mathrm{CT}^{2}$ et ont fait l'objet d'un suivi longitudinal, a montré que l'activité métabolique au repos de l'amygdale cérébrale était associée de façon indépendante au risque de survenue d'une cardiopathie ischémique, et qu'en raison de l'implication de l'activité de l'amygdale dans l'activité de la moelle osseuse et de l'inflammation artérielle, celle-ci jouait un rôle de médiation important entre l'activité de l'amygdale et la pathologie cardiovasculaire [19].

\section{Conclusion}

Diverses circonstances rencontrées en milieu de travail concourent à soumettre de très nombreuses personnes à un risque cardiovasculaire élevé. La prévention du stress professionnel relève bien entendu avant tout du médecin du travail, et de nombreuses initiatives ont été prises ces dernières années pour identifier et réduire les «risques psychosociaux» (RPS dans le jargon d'aujourd'hui). Mais le médecin traitant et le cardiologue ont eux aussi un rôle important à jouer vis-à-vis de leurs patients en prenant en compte leurs conditions de travail, et en attirant leur attention sur leur potentiel rôle néfaste, comme sur celui des autres facteurs de risque professionnels de pathologies cardiovasculaires. Le stress professionnel chronique a d'ailleurs été clairement reconnu, grâce notamment aux travaux du consortium IPDWork, comme un facteur de risque qui doit être pris en compte dans la pratique clinique par les European Guidelines on Cardiovascular Disease Prevention in Clinical Practice publiés en 2016 [20]. $\diamond$

\section{SUMMARY}

\section{Stressful work and heart do not get along well}

As other occupational risk factors, like noise, heat or some chemicals, stress at work may induce coronary heart disease. Occupational stress has several components and is usually measured using two different instruments: the Karasek model defines the job strain as the association between high constraints and low latitude at work, whereas the Siegrist model relies on the imbalance between efforts at work and rewards. It is now well established that occupational stress is an

\footnotetext{
${ }^{2}$ La TEP-TDM (Petscan ou PET-CT) est une technique d'imagerie hybride associant une caméra, le tomographe par émission de positons (TEP), à un scanner multibarette (tomodensitométrie ou TDM). La TEP mesure la distribution sur l'ensemble du corps d'une molécule marquée par un émetteur de positons. Une acquisition d'images est effectuée pour chaque modalité, suivie d'une fusion de ces images TEP et TDM. Cela permet une meilleure qualité d'imagerie, mais aussi un repérage anatomique précis des lésions métaboliques dans les trois plans de l'espace. La TEP utilise en clinique courante un seul traceur, le fluorodéoxyglucose marqué au fluor 18 (18F) (FDG). Celui-ci permet la détection des lésions hypermétaboliques, en particulier néoplasiques.
} 
independent risk factor of coronary heart disease, the attributable risk fraction being about $3.5 \%$, yielding some $3,400-4,000$ incident cases each year in France. Several biological mechanisms have been hypothesized to explain how stress can induce coronary heart disease, but this remains largely unknown. $\diamond$

\section{LIENS D'INTÉRÊT}

Les auteurs déclarent n'avoir aucun lien d'intérêt concernant les données publiées dans cet article.

\section{RéFÉRENCES}

1. Virkkunen H, Härmä M, Kauppinen T, et al. Shift work, occupational noise and physical workload with ensuing development of blood pressure and their joint effect on the risk of coronary heart disease. Scand J Work Environ Health 2007 ; 33 : 425-34.

2. Roseman KD. Cardiovascular disease and environmental exposure. Br J Ind Med 1979 ; 36 : 85-97.

3. Soteriades ES, Smith DL, Tsismenakis AJ, et al. Cardiovascular disease in US firefighters: a systematic review. Cardiol Rev $2011 ; 19$ : 202-15.

4. Virtanen $M$, Heikkila K, Jokela $M$, et al. Long working hours and coronary heart disease: a systematic review and meta-analysis. Am J Epidemiol 2012 ; 176 : 586-96.

5. Harari G, Green MS, Zelber-Sagi S. Combined association of occupational and leisure-time physical activity with all-cause and coronary heart disease mortality among a cohort of men followed-up for 22 years. Occup Environ Med 2015 ; 72 : 617-24.

6. Malard L, Chastang JF, Niedhammer I. Changes in psychosocial work factors in the French working population between 2006 and 2010. Int Arch Occup Environ Health 2015 ; 88 : 235-46.

7. Karasek R, Baker D, Marxer F, et al. Job decision latitude, job demands, and cardiovascular disease: a prospective study of Swedish men. Am J Public Health 1981 ; 71 : 694-705.

8. Siegrist J. Adverse health effects of high-effort/low-reward conditions. J Occup Health Psychol 1996; $1: 27-41$.

9. Niedhammer I, Chastang JF, Gendrey L, et al. Propriétés psychométriques de la version française des échelles de la demande psychologique, de la latitude décisionnelle et du soutien social du Job Content Questionnaire de Karasek : résultats de l'enquête nationale SUMER. Santé Publique $2006 ; 18$ : 413-27.

10. Niedhammer I, Siegrist J, Landre MF, et al. Étude des qualités psychométriques de la version française du modèle du Déséquilibre Efforts/Récompenses. Rev Epidemiol Sante Publ 2000 ; $48: 419-37$.
11. Hintsa T, Shipley MJ, Gimeno D, et al. Do pre-employment influences explain the association between psychosocial factors at work and coronary heart disease? The Whitehall II study. Occup Environ Med 2010 ; $67: 330-4$.

12. Niedhammer I, Malard L, Chastang JF. Occupational factors and subsequent major depressive and generalized anxiety disorders in the prospective French national SIP study. BMC Public Health. $2015 ; 15: 200$.

13. Bongers PM, Kremer AM, ter Laak J. Are psychosocial factors, risk factors for symptoms and signs of the shoulder, elbow, or hand/wrist? A review of the epidemiological literature. Am J Ind Med $2002 ; 41: 315-42$.

14. van der Doef M, Maes S. The job demand-control(-support) model and psychological well-being: a review of 20 years of empirical research. Work Stress $1999 ; 13: 87-114$.

15. Kivimäki M, Kawachi I. Work stress as a risk factor for cardiovascular disease. Curr Cardiol Rep $2015 ; 17: 630$.

16. Kivimaki M, Nyberg ST, Batty GD et al. Job strain as a risk factor for future coronary heart disease: collaborative analysis of 2358 events in 197,473 men and women. Lancet $2012 ; 380: 1491-7$.

17. Meneton P, Lemogne $C$, Herquelot $\varepsilon$, et al. Primary cardiovascular disease risk factors predicted by poor working conditions in the GAZEL cohort. Am J Epidemiol 2017. https://doi.org/10.1093/aje/kwx152.

18. Lemogne $C$, Meneton $P$, Wiernik $\varepsilon$, et al. When blue-collars feel blue: depression and low occupational grade as synergistic predictors of incident coronary heart disease in middle-aged working individuals. Circ Cardiovasc Qual Outcomes 2017 ; 10 : pii: e002767.

19. Tawakol A, Ishai A, Takx RA et al. Relation between resting amygdalar activity and cardiovascular events: a longitudinal and cohort study. Lancet 2017 ; $389: 834-45$.

20. 2016 European guidelines on cardiovascular disease prevention in clinical practice. The sixth joint task force of the European Society of Cardiology and other societies on cardiovascular disease prevention in clinical practice. Eur Heart J 2016 ; 37 : 2315-81.

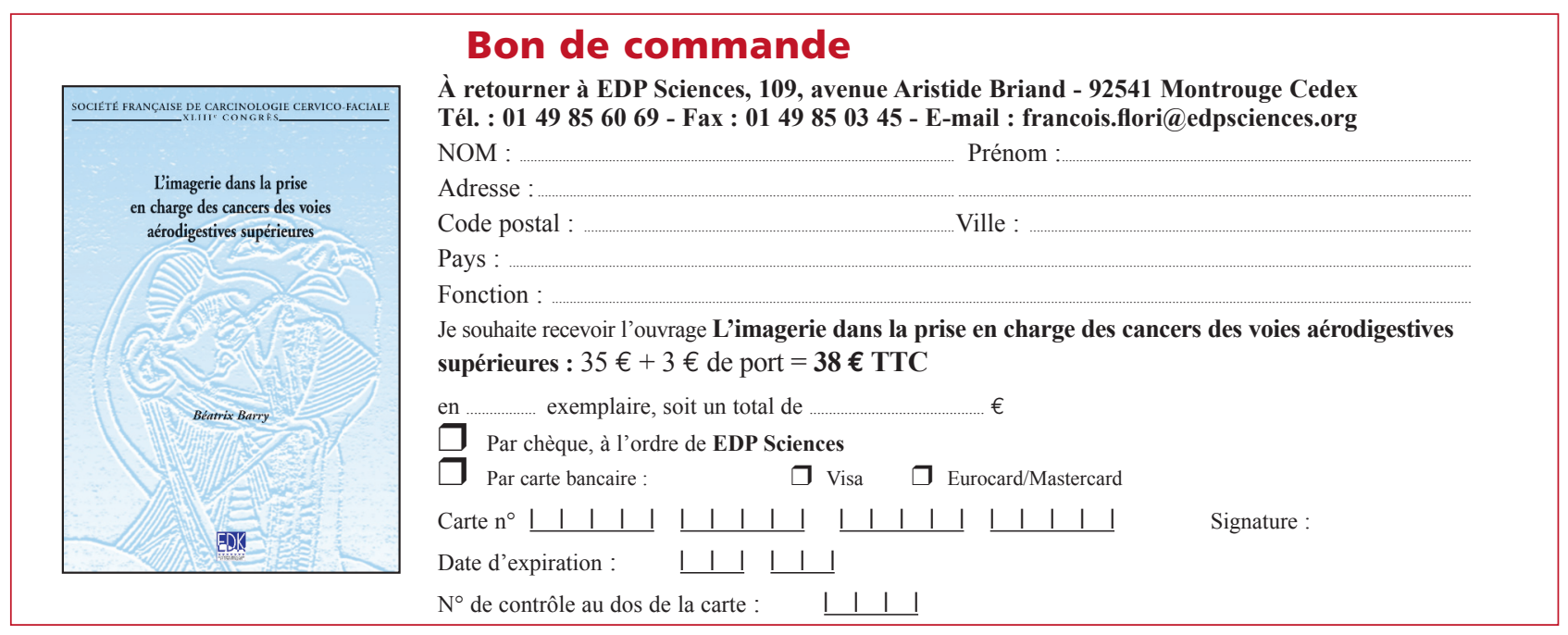

TIRÉS À PART

M. Goldberg

\section{Bon de commande}

À retourner à EDP Sciences, 109, avenue Aristide Briand - 92541 Montrouge Cedex Tél. : 0149856069 - Fax : 0149850345 - E-mail : francois.flori@edpsciences.org NOM : Prénom

Code postal : Ville :

Pays :

Fonction :

Je souhaite recevoir l'ouvrage L'imagerie dans la prise en charge des cancers des voies aérodigestives supérieures : $35 €+3 €$ de port $=\mathbf{3 8} €$ TTC

en ................. exemplaire, soit un total de ..........................................

$\square$ Par chèque, à l'ordre de EDP Sciences

Par carte bancaire : $\square$ Visa $\square$ Eurocard/Mastercard

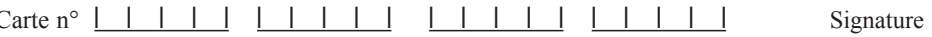

Date d'expiration: $\quad$ | | | | 1 | |

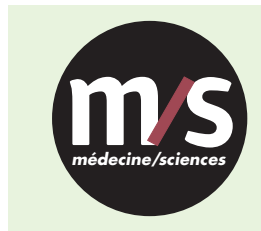

Tarifs d'abonnement $\mathrm{m} / \mathrm{s}-2017$

$>$ Grâce à $m / s$, vivez en direct les progrès

des sciences biologiques et médicales

Abonnez-vous

à médecine/sciences

Bulletin d'abonnement page 1010 dans ce numéro de $\mathrm{m} / \mathrm{s}$

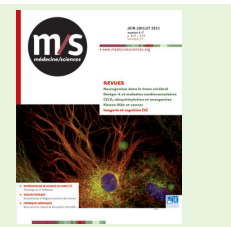

